\section{Needing more: the case for extra high compression for tall men in UK leg ulcer management}

\section{Alison Hopkins, Richard Bull, Fran Worboys}

Accelerate CIC, Mile End Hospital, Bancroft Road, London, UK

\section{Introduction}

This paper provides an observation on the approach taken to the use of compression therapy in the UK within the context of patient and expert experience. Venous ulceration is relatively common affecting 1$3 \%$ of the population; ${ }^{1,2}$ it is a treatable condition but chronicity or non-healing is commonplace. Many patients will have a difficult experience often because compression is little understood in practice and usage across the UK is generally inadequate with pockets of good practice across the country. This paper contends that UK guidance offers little hope of a therapeutic intervention for the complex or non-standard group; there is no 'Plan B' if the patient does not respond favourably to standard UK high compression. The authors present 2 patients that are examples of this issue and have observed that tall men require compression greater than the UK standard; unfortunately the provision of strong compression of $>60$ $\mathrm{mmHg}$ is not promoted within the UK.

\section{Variations in guidance}

The UK national guidance ${ }^{1}$ for the man- agement of venous ulceration is weekly compression therapy where high compression is stated as being $23-35 \mathrm{mmHg}$; in practice clinicians and industry refer to meeting $40 \mathrm{mmHg}$ at the gaiter. This is considered gold standard therapy within the UK. However, there are international variations $^{3}$ and these are that compression is described as: i) mild $<20 \mathrm{mmHg}$; ii) moderate $20-40 \mathrm{mmHg}$; iii) strong $40-60 \mathrm{mmHg}$; iv) very strong $>60 \mathrm{mmHg}$.

Specialist clinicians in the UK do not promote the use of compression above 40 $\mathrm{mmHg}$; there is the assumption that this is already high compression and is the upper limit. With the exception of the authors own training materials, there is no evidence that even within specialist leg ulcer modules that compression above $40 \mathrm{mmHg}$ is promoted; there is plenty of anecdotal evidence that the UK consensus is that the application of strong compression is simply too strong and is seen as too high a risk for general leg ulcer management. Clinicians are advised $^{1}$ on the areas that need to be considered when determining the strength of the compression therapy, such as ankle width, underlying arterial status or tolerance and pain management. The standard care is promoted and is considered key to the provision of optimal care and a quality intervention. However this intervention assumes that patients are similar, require the same level of compression therapy for this to be effective and also that nursing intervention is also standard. This is clearly not the case. Also guidance states that there are a number of parameters (pain, arterial status) that can be reviewed in order to provide safe but reduced compression. However only in the presence of a larger ankle circumference is the clinician advised to increase the level of compression to accommodate this feature and thereby providing a higher sub-bandage pressure and thus a therapeutic intervention.
Correspondence: Alison Hopkins, Accelerate CIC, Mile End Hospital, Bancroft Road, London E1 4DG, UK.

E- mail: alison.hopkins2@nhs.net

This work is licensed under a Creative Commons Attribution 4.0 License (by-nc 4.0).

(C) Copyright A. Hopkins et al., 2017

Licensee PAGEPress, Italy

Veins and Lymphatics 2017; 6:6630

doi:10.4081/vl.2017.6630

\section{When an ulcer fails to respond to compression therapy}

When the use of optimal compression therapy at around $35 \mathrm{mmHg}$ is failing to heal the leg ulcer, the guidance for the clinician is to use advanced dressings. If the compression therapy is not being tolerated by the patient, the guidance is to increase compression tolerance through patient education and adjustment and/or reduction of the compression level; there is the underlying belief that light compression is better than nothing. There is no guidance to review the therapeutic value of this potent intervention. It is not routine practice to question whether the standard high compression is actually adequate for that patient. There is no recommendation that improving the bandaging technique or consistency of intervention may increase its efficacy or tolerance; conversely patient experience would recognise the wide variations in application techniques. There is thus no suggestion that the patient may benefit from a higher level of compression.

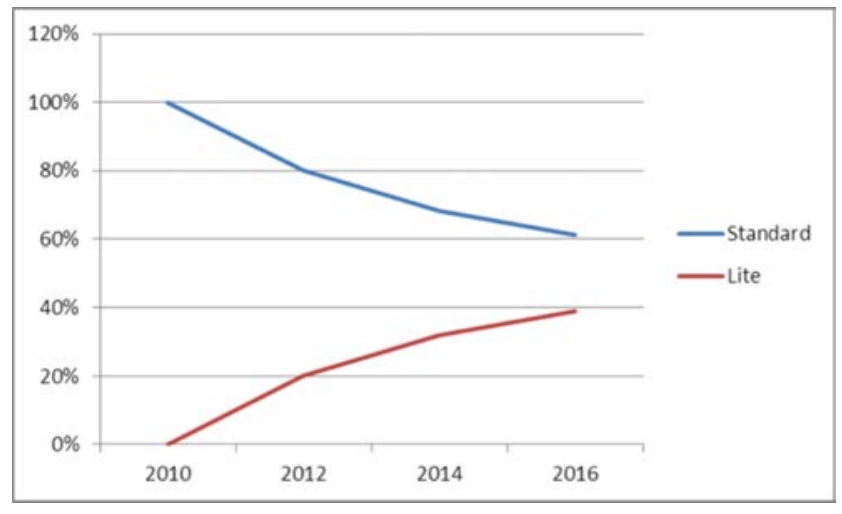

Figure 1. National sales of a Multilayer compression regime.

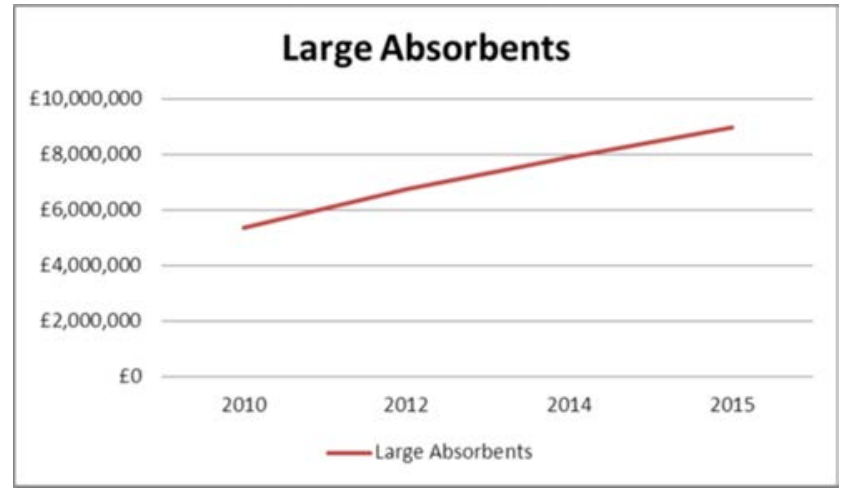

Figure 2. Large absorbents. 


\section{The UK picture}

There is a growing gap between the theory of compression therapy as a clinical intervention and the UK practice of application. Compression is increasingly being taught as a task and not as a therapeutic intervention, often due to workforce capacity issues and the lack of available time for adequate training.

The association of poor compression use with limb amputation has led to a risk averse approach to compression use and a culture of fear within nursing. This has meant a reduction in use of standard compression of $40 \mathrm{mmHg}$ and a promotion of light or reduced compression in the UK; this can only lead to an increasing number of patients receiving inadequate compression therapy and there is certainly enough anecdotal evidence to support this.

The default to light compression is becoming the norm in clinical practice; a recent report of a leg ulcer audit found that of those who would benefit from compression therapy, $16 \%$ were in high compression and $30 \%$ were in light compression. ${ }^{4}$ Figure 1 demonstrates the growing use of reduced compression within one well known multilayer compression regime that has two versions: standard high compression and a reduced or light compression. It appears that the introduction of the light compression version was in response to nursing requests and the belief that patients do not tolerate high compression therapy. This assumption needs to be challenged. The authors and those clinicians who attend the ICC would refute this, noting that lack of patient tolerance is predominantly down to nursing technique, skill and knowledge of this potent therapy.

The impact of non-therapeutic care is significant, resulting in poor oedema and exudate management in both leg ulcers and lymphorrhea; alongside this escalation of light compression we are seeing an increase in spend on extra-large superabsorbent dressings (a pad size greater than $15 \times 15$ $\mathrm{cm}$ ) that simply absorbs this unmanaged exudate (Figure 2). Unfortunately admission for cellulitis due to erosive or leg ulceration are common.

\section{The impact of height}

UK guidance ${ }^{1}$ does not consider the impact of height on the level of compression a patient requires for it to be therapeutic. This paper contends that tall men are a group of patients who do not respond to standard compression but experience a destructive response to its use (Figure 3). It is clear that hydrostatic pressure increases with height and that ordinarily 60-90 $\mathrm{mmHg}$ is required to narrow and occlude

the veins. It is likely that a resting pressure of greater than $60 \mathrm{mmHg}$ is thus required for vein occlusion in taller men. However, in the UK where multicomponent elastic

Table 1. John and Geoff's common experience.

No post thrombotic history, duplex discounted the presence of Venous reflux

Young and previously active (50 and 40 years)

Variety of Standard compression systems tried (UK High)

Compression was not tolerated despite opioid use

Destructive cycle of pain, oedema and exudate

Labelled as rare and diagnosis unclear despite negative biopsies

Each responded swiftly to Strong compression with a resting pressure of $>60 \mathrm{mmHg}$; no medical intervention was required

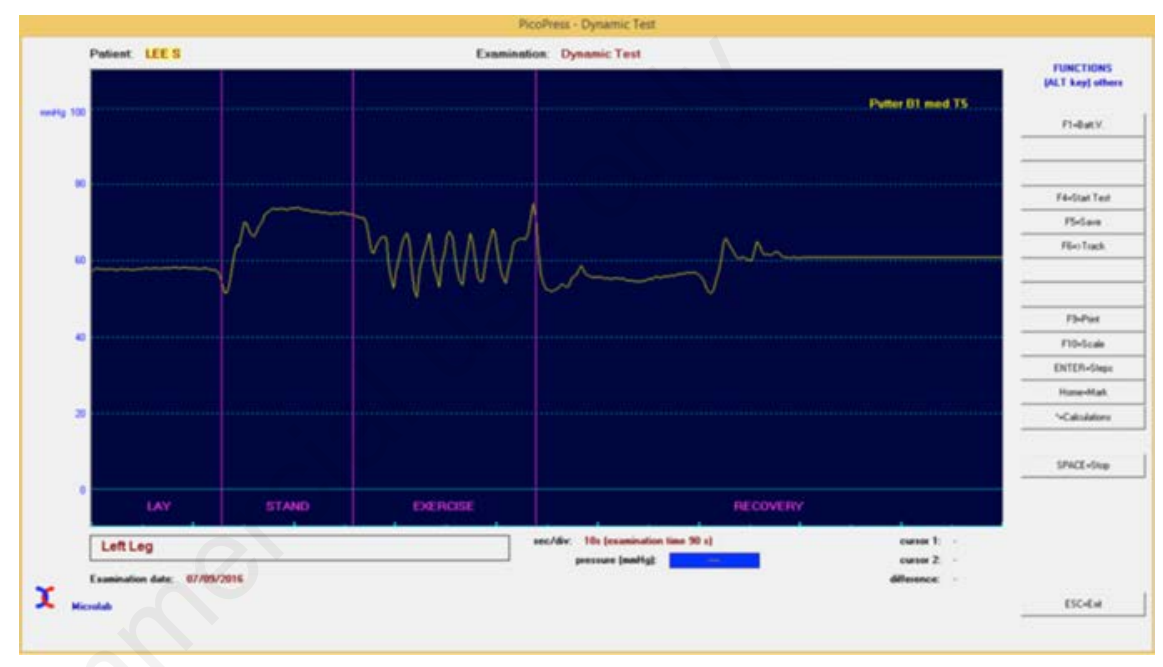

Figure 3. Inelastic multilayer regime.

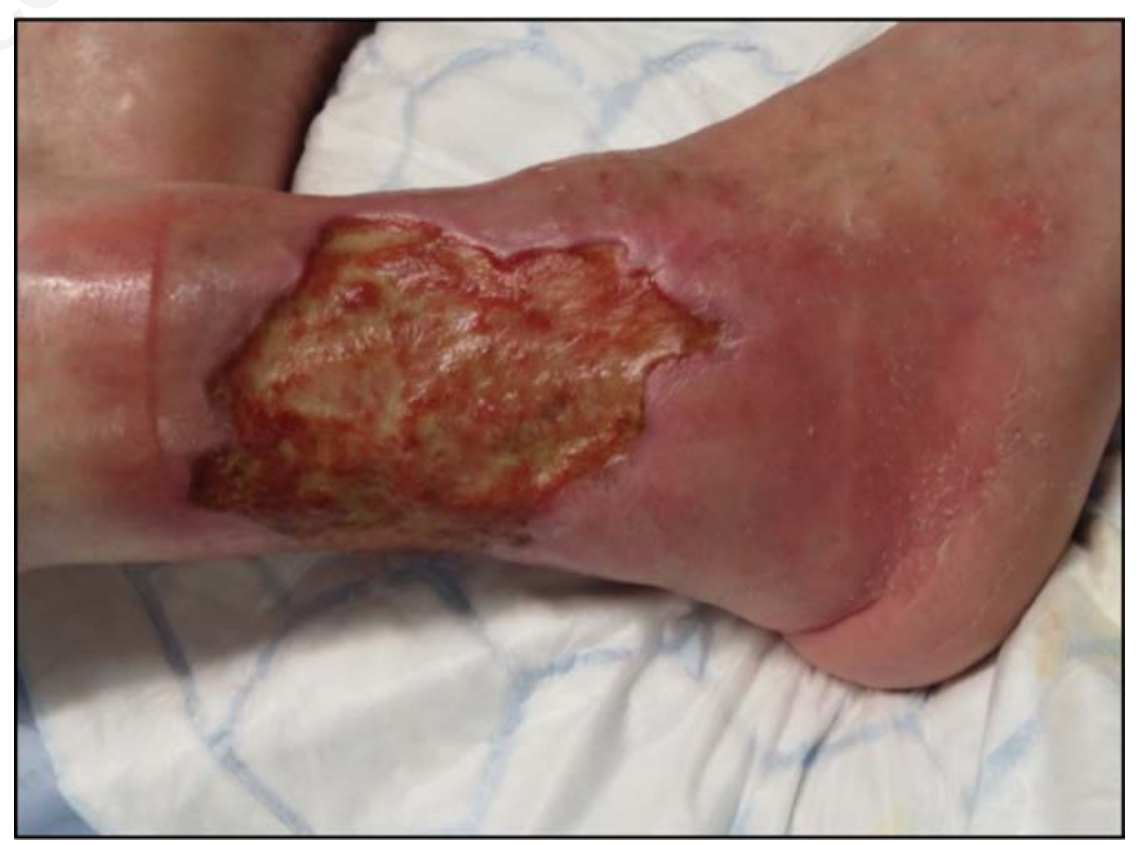

Figure 4. John's ulcer, right lower gaiter present for 4 years. 
compression is common and the role of static stiffness is not appreciated, this level will rarely be achieved and is certainly not promoted.

John and Geoff were referred to the author's Treatment Centre for assessment following significant interventions from Dermatology and Vascular departments (Table 1). Their experience of cycles of improvement, deterioration, referral to various clinicians and query over their diagnosis all took its toll on their lives. Their journeys and the impact of sustained very strong compression has led us to question the gaps in current UK guidance.

It is the authors' observation that the standard high compression, ${ }^{1}$ in the presence of height greater than $180 \mathrm{~cm}$, acted as reduced or light compression; this generated a destructive cycle of uncontrolled oedema, causing exudate and further erosions, additional pain and lack of tolerance for an ineffective regime. The patient's pain also caused concern and the nurses reduced the level of compression further by applying light compression regimes. As described previously, guidelines advise the clinicians to seek alternative diagnosis or treatments; unfortunately these simply prolonged the patient's deterioration and intractable pain.

The clinical goal was to increase the level of compression to therapeutic levels alongside the provision of adequate analgesia; an inelastic multilayer regime was used. This ensured that the hydrostatic nature of the ulceration or functional venous disease was managed, delivering circa $60 \mathrm{mmHg}$ resting pressures at $\mathrm{B} 1$ level (Figure 3) and towards $70 \mathrm{mmHg}$ working pressure. This approach led to healing without any additional medical interventions; management was mostly a straightforward therapeutic task once the correct level of compression was reached. It is also important to note that this strong compression helped to reduce the pain despite the fear that this would exacerbate it; their experience was that their legs felt relief at the extra stiffness this regime provided, creating confidence in this approach (Figures 4-7).

\section{Conclusions}

This brief paper has identified some of the inadequacies of the current UK guidance on the management of venous ulcers namely what constitutes high compression and the limited advice for a non-standard patient. Guidelines and current UK education promote a risk aversion approach thereby limiting the treatment options for the most complex of patients.

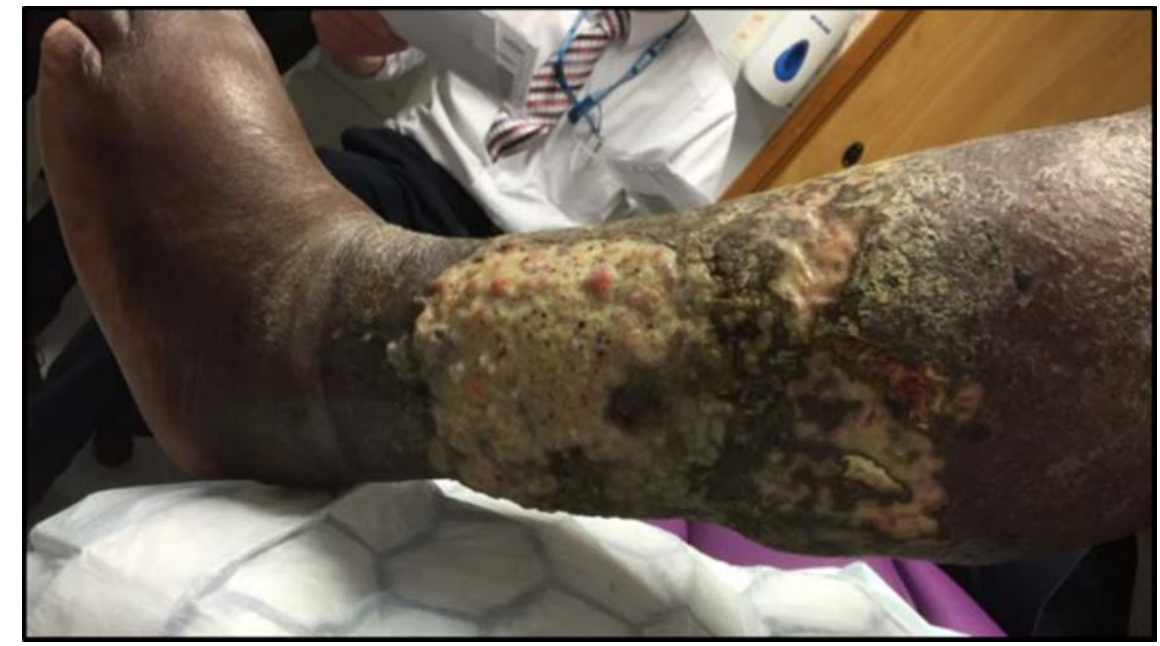

Figure 5. Geoff's left leg ulceration, present for 18 months.

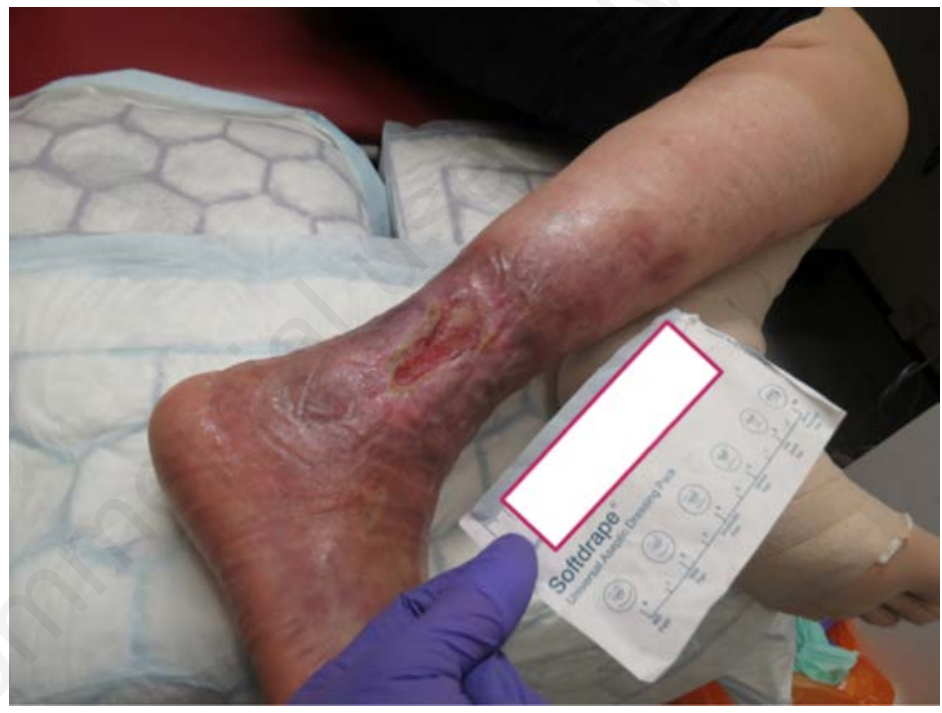

Figure 6. John's ulcer 12 months later.

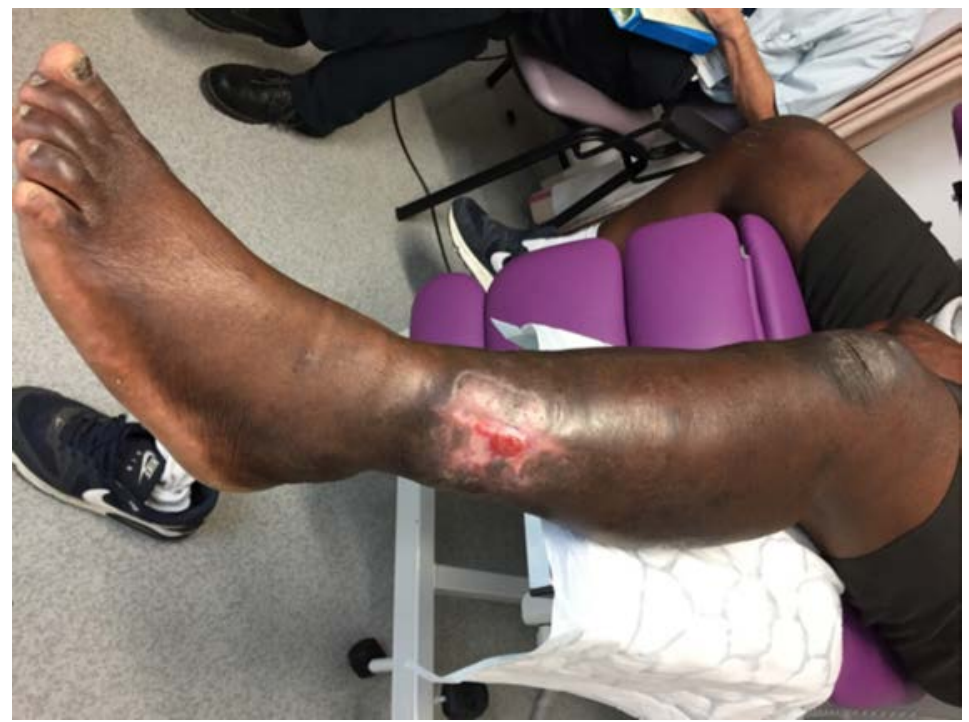

Figure 7. Geoff's ulcer 9 months later. 
This paper introduces 2 patients for whom their destructive ulceration was reversed using extra high or strong compression, an approach that would be considered dangerous in the UK. The authors contend that strong compression addresses the larger hydrostatic column in taller people. However, it is unclear whether bandages also behave differently in tall people.

The use of light compression is on the increase in the UK and this can only have a detrimental impact on patients' lives, the nursing workforce and the health economy. Clinicians in the UK and the ICC need to critique the current status of leg ulcer management and promote the international consensus opinion of what constitutes high and thus therapeutic compression.

\section{Learning points}

- Clinicians in the UK need to question the level of sub-bandage pressure required in the non-healing group.

- That taller patients require strong or very strong compression and this is above the UK recommendations.

- That contrary to popular belief, strong compression can significantly reduce pain in the larger limb.

\section{References}

1. Scottish Intercollegiate Guidelines Network (SIGN). Management of chronic venous leg ulcers. Clinical guideline No. 120. Scottish Intercollegiate Guidelines Network; 2010. Available from: www.sign.ac.uk

2. Kunimoto B, Cooling M, Gulliver W, et al. Best practices for the prevention and treatment of venous leg ulcers. Ostomy Wound Management 2001;47:34-50.

3. World Union of Wound Healing Societies (WUWHS). Principles of best practice: compression in venous leg ulcers. A consensus document. London: MEP Ltd.; 2008. Available from: http://www.woundsinternational.com/m edia/issues/65/files/content_25.pdf

4. King BM. Leg ulcer audit report. Leg Ulcer Forum J 2016;28:16-9. 